\title{
LYMPHOCYTE FUNCTION-ASSOCIATED ANTIGEN 1 OVEREXPRESSION AND T CELL AUTOREACTIVITY
}

\author{
BRUCE RICHARDSON, DANIEL POWERS, FORREST HOOPER, RAYMOND L. YUNG, and \\ KENNETH O'ROURKE
}

\begin{abstract}
Objective. To determine if DNA methylation inhibitors make $T$ cells autoreactive by inducing lymphocyte function-associated antigen type 1 (LFA-1) (CD11a/ CD18) overexpression.

Methods. T cell clones were treated with 3 distinct DNA methylation inhibitors or were stably transfected with a CD18 cDNA in a mammalian expression vector, and the effects on LFA-1 expression and activation requirements were examined.

Results. LFA-1 overexpression, caused by DNA methylation inhibitors or by transfection, correlates with the development of autoreactivity.

Conclusion. LFA-1 overexpression may contribute to $T$ cell autoreactivity.

Investigators at this laboratory have reported that human and murine cloned, antigen-specific, $\mathrm{CD} 4+\mathrm{T}$ cells become autoreactive following treatment with DNA methylation inhibitors like 5-azacytidine (5-azaC) and procainamide (PCA) (1-3). The autoreactivity has pathologic significance, because $T$ cells made autoreactive with these drugs are sufficient to cause a lupus-like disease when injected into syngeneic mice (3). This system is relevant to human lupus, because PCA induces a lupus-like disease in patients receiving this drug (4), and because patients with idiopathic lupus have
\end{abstract}

Supported by Public Health Service grants 2-P60-AR20557, F32-AI-08253, and R01-AI-25526, and a Merit Review grant from the Veterans Administration.

Bruce Richardson MD, PhD: University of Michigan, Ann Arbor, and the Ann Arbor Veterans Administration Hospital; Daniel Powers, MS: University of Michigan; Forrest Hooper, BA: University of Michigan; Raymond L. Yung, MD: University of Michigan; Kenneth O'Rourke, MD: Bowman Gray School of Medicine, Winston-Salem, North Carolina.

Address reprint requests to Bruce Richardson $\mathrm{MD}, \mathrm{PhD}$, R4540 Kresge 1, Ann Arbor, MI 48109-0531.

Submitted for publication September 21, 1993; accepted in revised form March 29, 1994. impaired T cell DNA methylation (5), similar to cells treated with 5-azaC or PCA. How DNA methylation inhibitors modify $\mathrm{T}$ cells to induce autoreactivity is, however, unknown.

DNA methylation is one of the mechanisms regulating gene expression. In genes regulated by such mechanisms, hypomethylation of regulatory sequences correlates with active transcription, while methylation of the regulatory sequences correlates with transcriptional suppression (6). We have previously shown that concentrations of 5 -azaC which induce autoreactivity also modify $\mathrm{T}$ cell gene expression $(7,8)$, and proposed that alterations in the expression of one or more $T$ cell genes may be responsible for the autoreactivity. An extensive search demonstrated that the most reproducible change induced by 5 -azaC was an increase in cell surface leukocyte functionassociated antigen 1 (LFA-1) (CD11a/CD18) (8). Furthermore, small amounts of anti-CD11a completely inhibited the associated autoreactive response, while significantly greater amounts were required to inhibit the antigen response of the same cells (8). This suggested that increased LFA-1 expression might contribute to autoreactivity, and anti-LFA-1 reverse it. However, it is also possible that other, as-yet-undetected gene products are responsible for the autoreactivity, or that the increase in LFA-1 expression does not directly cause the autoreactivity.

In this report we have further examined the role of LFA-1 overexpression in $T$ cell autoreactivity. First, the correlation between increased LFA-1 expression and autoreactivity was confirmed using additional, mechanistically distinct DNA methylation inhibitors. Second, LFA-1 expression was increased by transfection of $T$ cell clones, and effects on $T$ cell activation requirements were examined. The results support the hypothesis that LFA-1 overexpression contributes to $T$ cell autoreactivity. 


\section{MATERIALS AND METHODS}

T cell lines. The human $T$ cell line Jurkat (E6-1) was kindly donated by Dr. Arthur Weiss (University of California at San Francisco) and cultured as previously described (2). The cloned, tetanus toxoid (TT)-reactive CD4+ human T cell lines, including TT18X, TT18I, TT37L, TT44G, and TT48C, and the cloned CD4+ conalbumin-reactive murine line D.10.G4.1, have been previously described, and all demonstrate similar autoreactivity when treated with DNA methylation inhibitors $(1-3,8)$.

All human lines were cloned from the same donor (BR) by limiting dilution using $\leq 0.2$ cells/well. Cells were maintained in interleukin-2 (IL-2) (supernatant from the MLA-144 T cell line [9])-containing media (RPMI 1640 supplemented with $10 \%$ fetal calf serum, penicillin, streptomycin, and L-glutamine) by periodic rechallenge with TT (1:50 dilution; Wyeth, Marietta, PA) and irradiated $(3,000 \mathrm{R})$ autologous peripheral blood mononuclear cells (PBMC) as antigen-presenting cells (APC) as described elsewhere $(1,2)$. Where indicated, the cells were treated with 5-azaC (Aldrich, Milwaukee, WI) or PCA (Sigma, St. Louis, MO). Phytohemagglutinin (PHA)-activated, IL-2-dependent CD4+ T cells were also generated as previously described (10).

Monoclonal antibodies and flow cytometric analysis. Anti-CD11a (TS 1/22, an inhibitor of T cell responses) (11) and anti-CD18 (TS 1/18) (11) were obtained from the American Type Culture Collection (Rockville, MD) and maintained according to instructions. The anti-CD1la monoclonal TA-1, which does not inhibit $\mathrm{T}$ cell responses (12), was kindly donated by Dr. Tucker LeBien. Anti-CD2-RD1 and anti-CD3-RD1 were obtained from Coulter (Hialeah, FL), and fluorescein isothiocyanate-conjugated goat anti-mouse Ig was obtained from Sigma.

Direct and indirect immunofluorescence staining were performed as previously described $(1,7,8)$, and fluorescence was analyzed using a Coulter EPICS or ELITE flow cytometer in 1- or 2-color mode. When data from the EPICS were used (reported as log fluorescence intensity), relative fluorescence intensity (RFI) between markers was calculated using the formula: $\log \mathrm{RFI}=[(\mathrm{A}-\mathrm{B}) / 25.2] \log _{2}$, where $A$ and $B$ represent the mean channel fluorescence or peak fluorescence of 2 markers. When the ELITE data were used (reported as linear fluorescence intensity), RFI could be simply calculated as the difference in intensity. Cell cycle analysis was performed using propidium iodide as previously described $(3,8)$.

Ultraviolet (UV) light exposure. Human T cells were suspended in $2 \mathrm{~mm}$ of phosphate buffered saline (PBS) in uncovered 6-cm petri dishes and exposed to light from a bank of 6 Westinghouse FS-40 lamps for varying lengths of time. The flux of light $\left(\mathrm{J} / \mathrm{m}^{2}\right)$ was measured by an externally calibrated LM HO6 radiometer (International Light, Newburyport, MA). Where indicated, wavelengths $<335 \mathrm{~nm}$ were absorbed using a filter (Schott Glass Technologies, Duryea, PA). When the filter was used, exposure times were lengthened by a factor of 2.57 to assure that the same amount of light energy $\left(\mathrm{J} / \mathrm{m}^{2}\right.$, as measured by the radiometer) was applied to the cells.

Proliferation assays. Proliferation assays were performed as previously described $(2,3)$. Briefly, IL-2- dependent T cells were washed, then cultured in $200 \mu \mathrm{l}$ of medium lacking IL-2, together with irradiated PBMC as APC. TT was added where indicated. Controls always included T cells and PBMC cultured alone. Proliferation was measured 3 days later by tritiated thymidine (New England Nuclear, Boston, MA) incorporation (1-3).

DNA deoxymethylcytosine content. DNA was isolated and digested with DNase I, phosphodiesterase, and alkaline phosphatase (Sigma) as previously described $(2,5)$. The resulting nucleosides were supplemented with fluorodeoxyuracil (FdU; Sigma) as an internal standard, then separated by chromatography on a Beckman (San Ramon, CA) Ultrasphere $\mathrm{C} 18$ column $(4.6 \mathrm{~mm} \times 25 \mathrm{~cm})$, using a mobile phase consisting of $2-3 \%$ methanol in $0.01 M$ sodium acetate, $\mathrm{pH}$ 3.5 as described (5). Eluted material was detected using a Waters 990 diode array detector, recording UV spectra from 220-320 nm every 100 milliseconds. Integrations were performed at $300 \mathrm{~nm}$, and quantitated relative to standard curves generated from purified commercial deoxycytosine $(\mathrm{dC})$ and deoxymethylcytosine $\left(\mathrm{d}^{\mathrm{m}} \mathrm{C}\right)$, both obtained from Sigma. Results are expressed as $\% \mathrm{~d}^{\mathrm{m}} \mathrm{C}=\left[\mathrm{d}^{\mathrm{m}} \mathrm{C} /\left(\mathrm{d}^{\mathrm{m}} \mathrm{C}+\mathrm{dC}\right)\right]$ $\times 100$, and all determinations were performed in duplicate.

Electroporation. Initial experiments demonstrated that $220 \mathrm{~V}$ and $960 \mu \mathrm{F}$ gave optimal chloramphenicol acetyltransferase (CAT) expression by a CAT-pSV2 construct in IL-2-dependent lines. However, CAT expression was low, suggesting relatively low transfection efficiency. Therefore, long-term, stable transfectants were used, rather than transient transfection systems. To generate the stable transfectants, a full-length human CD11a complementary DNA (cDNA) cloned into CDM8 (13), kindly donated by Dr. Tim Springer, and a full-length human CD18 cDNA cloned into pEMO, kindly donated by Dr. James Wilson (14), were used. Control vectors included CDM8 and pEMO without inserts. For transfection, the CDM8 constructs were linearized with Nhe I and the pEMO constructs with Nde I (both from Boehringer Mannheim, Indianapolis, IN). T cell clones were washed with PBS, and $19 \times 10^{6}$ were suspended in $1 \mathrm{ml}$ of sterile HEPES electroporation buffer ( $20 \mathrm{mM}$ HEPES, 137 $\mathrm{m} M \mathrm{NaCl}, 5 \mathrm{~m} M \mathrm{KCl}, 0.7 \mathrm{~m} M \mathrm{Na}_{2} \mathrm{HPO}_{4}, 6 \mathrm{~m} M$ dextrose, $\mathrm{pH}$ 7.05) in an electroporation cuvette. Linearized construct (11-15 $\mu \mathrm{g}$ ) was added, the mixture incubated at $4^{\circ} \mathrm{C}$ for 10 minutes, then electroporated at $220 \mathrm{~V}$ and $960 \mu \mathrm{F}$, using a Gene Pulsar with capacitance extender (Bio-Rad, Hercules, CA). The cells were again incubated at $4^{\circ} \mathrm{C}$ for 10 minutes, washed once, then cultured at $10^{6} / \mathrm{ml}$ in IL-2-containing media, using 24-well plates (Falcon; Becton Dickinson, Lincoln Park, NJ). The next day, $10^{6}$ irradiated autologous PBMC were added to each well, and the cells were maintained as long-term lines with IL-2 and periodic restimulation with irradiated autologous PBMC but without TT.

Southern analysis. Southern analysis was performed using a modification of previously published techniques (14). Briefly, purified T cell DNA was digested with either Sfi1 (Gibco-BRL, Gaithersburg, MD) and Pac1 (United States Biochemical, Cleveland OH) or Sfi, Pacl, and Kpnl (Boehringer Mannheim). Ten micrograms of digested DNA was fractionated on a $1 \%$ agarose gel at $20 \mathrm{~V}$ for 15 hours, then capillary-transferred to a MagnaGraph (Micro Separations, Westborough, MA) nylon membrane, according to the manufacturer's instructions. The membranes were hybridized 

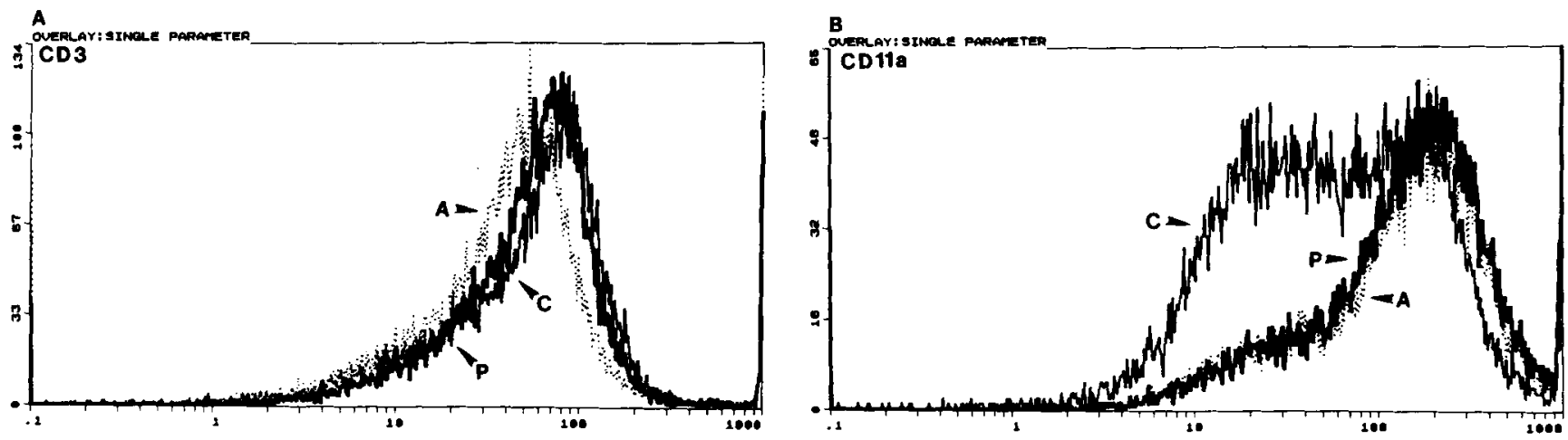

Figure 1. Effect of 5-azacytidine (5-azaC) and procainamide (PCA) on $\mathrm{T}$ cell expression of lymphocyte function-associated antigen 1 . A cloned CD4+ tetanus toxoid-reactive T cell line was treated with either $0.25 \mu M 5$-azaC or $50 \mu M$ PCA; 6 days later, treated and untreated cells were washed then stained with $\mathbf{A}$, anti-CD3 or B, anti-CD11a, then fuorescein isothiocyanate-conjugated goat anti-mouse Ig, and analyzed using a Coulter ELITE flow cytometer. CD3 staining of untreated (C; solid line), 5-azaC-treated (A; dotted line), and PCA-treated (P; heavy line) cells is approximately equal. CD11a staining of untreated cells (C; solid line) demonstrates a bimodal distribution, with peaks of approximately 20 and 150, while 5-azaC-treated (A; dotted line) and PCA-treated (P; heavy line) cells demonstrate nearly identical histograms, with a major peak at 200 , and a decrease in the former peak at 20 . X-axis represents fluorescence intensity in arbitrary units; $y$-axis represents cell number.

with ${ }^{32} \mathrm{P}$-labeled CD18 DNA fragments, cut as indicated in the text with Bam HI and Bst EII or Dpn I (Boehringer Mannheim), then washed, and autoradiograms were developed using previously described procedures $(8,15)$.

\section{RESULTS}

Initial experiments examined the proposed correlation between DNA hypomethylation, increased LFA-1 expression, and autoreactivity by comparing the effects of different DNA methylation inhibitors on $T$ cells. We chose 5-azaC, PCA, and UV light as agents which inhibit DNA methylation by different mechanisms. 5-azaC is incorporated into newly synthesized DNA where it covalently binds DNA MTase (16), while PCA binds and reversibly inhibits DNA MTase (17). The mechanism by which UV light inhibits DNA methylation is less clear, but presumably involves modification of cytosine residues by reactive oxygen species $(18,19)$.

Previous experiments demonstrated that 5-azaC and PCA inhibit T cell DNA methylation and induce autoreactivity (2), but LFA-1 expression was not examined in PCA-treated clones, and UV light was not tested. We treated human $\mathrm{T}$ cell clones with 5-azaC or PCA, and found that CD11a expression on a subset of the cells was increased $\sim 10$-fold (from 20 units to 200 units) (Figure 1), similar to 5-azaC-and PCA-treated polyclonal murine $\mathrm{T}$ cells (3). It should be noted that not all cells demonstrate an increase in CD11a expression. This is due in part to the fact that altered gene expression occurs, at best, in $50 \%$ of cells treated with DNA methylation inhibitors
(20), and in part to the percentage of cells in S phase at the time of treatment $(7,20)$. In 7 serial repeats of this experiment using 5 distinct cloned human CD4+ T cell lines, the average increase in CD11a expression over all the cells was $43 \pm 16 \%$ (mean \pm SEM; $P<0.05$, by univariate $t$-test for the mean), with a range of $14-136 \%$, similar to that previously found using 5-azaC (8).

To further confirm and extend these results, the cloned, conalbumin-reactive murine $\mathrm{T}$ cell line D.10.G4.1 was subcloned at 0.2 cells/well, and the subclone treated with $25 \mu M$ PCA. Similar results (a $76 \%$ increase in CD1 la) were observed. This increase also correlated with an increase in autoreactivity (mean \pm SEM 1,484 \pm 111 versus $11,920 \pm 710$ counts per minute, untreated versus treated, response of 20,000 $T$ cells to 20,000 irradiated syngeneic splenocytes).

Since the level of LFA-1 expression is in part dependent on $T$ cell activation (21), it is possible that the increase in LFA-1 is due to changes in cell cycle kinetics. Therefore, the effect of PCA on cell cycling was examined. TT44G cells were treated with $50 \mu M$ PCA, and 6 days later the percentages of cells in $G_{0} G_{1}$, $S$, and $G_{2} M$ phases were determined using propidium iodide staining and flow cytometric analysis. Similar to previous experiments using murine $\mathrm{T}$ cells (3), there was no significant effect of PCA on $\mathrm{T}$ cell cycle kinetics $(72 \pm 9 \%$ versus $62 \pm 4 \%, 15 \pm 6 \%$ versus 21 $\pm 2 \%$, and $14 \pm 4 \%$ versus $17 \pm 1 \%$, untreated versus treated, $\mathrm{G}_{0} \mathrm{G}_{1}, \mathrm{~S}$, and $\mathrm{G}_{2} \mathrm{M}$, respectively, mean $\pm \mathrm{SD}$ of 2 determinations). It remains possible that the 6-day drug treatment selects a subset with high LFA-1 

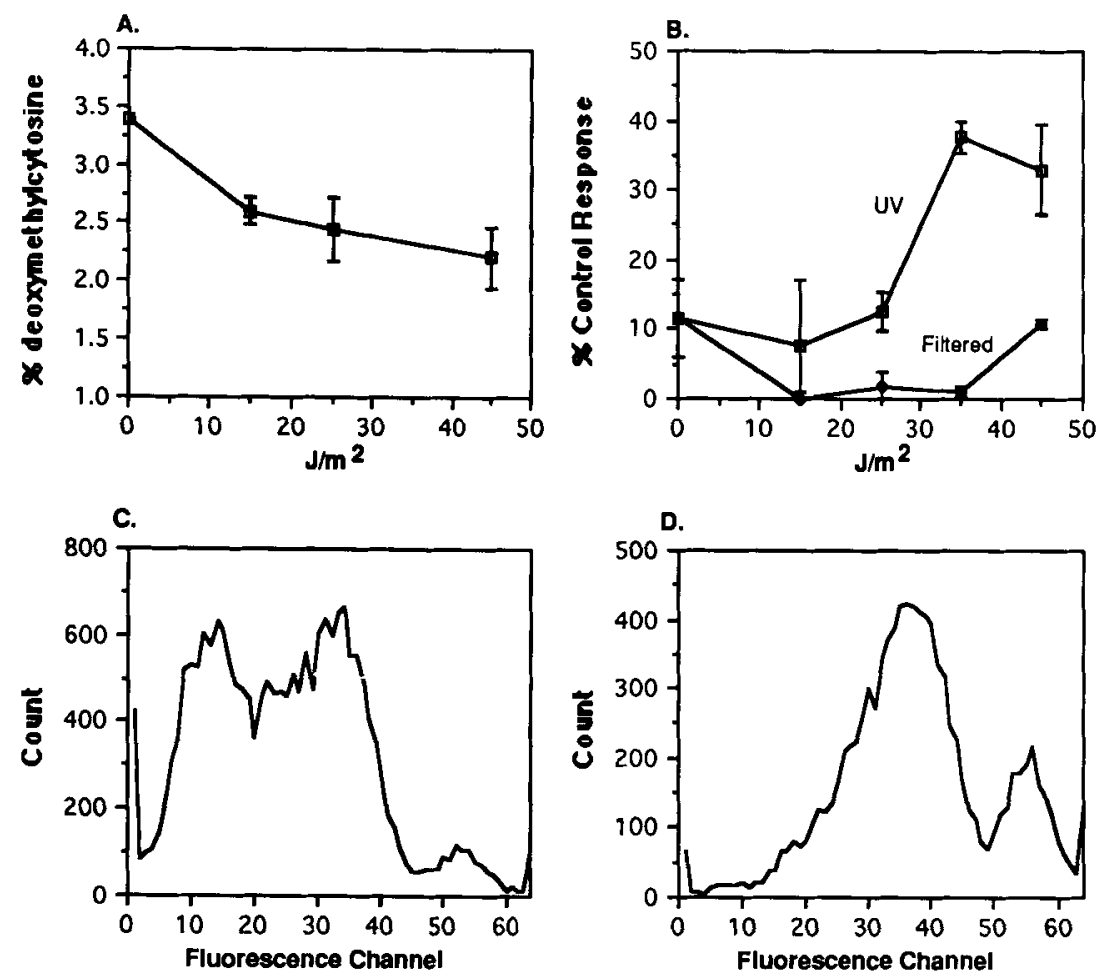

Figure 2. Effect of ultraviolet (UV) light on T cell DNA methylation (A), autoreactivity (B), and CD11a expression (C and D). A, Jurkat cells were exposed to the indicated amounts of UV-enriched light. Three days later, total $\mathrm{dC}$ and $\mathrm{d}^{\mathrm{m}} \mathrm{C}$ content was measured by reverse-phase high-performance liquid chromatography. Values are the $\% \mathrm{~d}^{\mathrm{m}} \mathrm{C}$ relative to total DNA $\mathrm{dC}$, and are the mean $\pm \mathrm{SEM}$ of 3 independent experiments, each performed in duplicate $\left(P<0.02\right.$, untreated versus $\left.45 \mathrm{~J} / \mathrm{m}^{2}\right)$. As a control, the experiments were repeated using a filter absorbing wavelengths shorter than $335 \mathrm{~nm}$ to delete UVB wavelengths, and exposure times were lengthened by a factor of 2.57 to maintain an equal amount of energy applied to the cells. With the filter, an exposure of $45 \mathrm{~J} / \mathrm{m}^{2}$ gave no significant decrease $(1.4 \pm 1.6 \%$ of control; $n$ $=3$ experiments) in $\mathrm{d}^{\mathrm{m}} \mathrm{C}$ content $(P<0.02$ relative to the same amount of unfiltered light $)$. B, Phytohemagglutinin-activated interleukin-2 (IL-2)-dependent CD4 + T cells were treated with the indicated amounts of UV-containing light, with or without a 335-nm filter. Cells were maintained in IL-2-containing media, and 6 days later, 20,000 cells were challenged with $10^{5}$ autologous irradiated antigen-presenting cells (APC), with or without $10 \mathrm{ng} / \mathrm{ml}$ anti-CD3 as a positive control. Proliferation was measured 3 days later by incorporation of ${ }^{3} \mathrm{H}$-thymidine. Values are the response of the cells to APC alone relative to the response to APC plus anti-CD3 $\left(P<0.02\right.$, untreated versus $\left.45 \mathrm{~J} / \mathrm{m}^{2}\right)$, and are the mean \pm SEM of quadruplicate determinations. The anti-CD3 response was similar for all light exposures, averaging $13,464 \pm 1,933 \mathrm{cpm}$. C, Tetanus toxoid-reactive T cell clones were exposed to UV light, and 6 days later, stained with anti-CD11a and fluorescein isothiocyanateconjugated goat anti-mouse Ig, then analyzed by cytofluorography using a Coulter EPICS C. X-axis represents log green fluorescence plotted as a 64-channel histogram; $y$-axis represents cell number. Unexposed cells are shown. D. The same cells as in $\mathbf{C}$ were exposed to $35 \mathrm{~J} / \mathrm{m}^{2}$ of UV-containing light, then analyzed by cytofluorography as in C. Controls using the 335 -nm filter showed no increase in expression of lymphocyte function-associated antigen 1 .

expression. However, the reproducibility of this observation on 6 distinct cloned lines is evidence for a direct effect on LFA-1 expression, rather than a lowexpressing subset contaminating all 6 lines.

The effects of UV light on T cell DNA methylation were examined by exposing Jurkat cells to varying amounts of UV-containing light, with or without a filter excluding wavelengths less than $335 \mathrm{~nm}$, then measuring total DNA $\mathrm{dC}$ and $\mathrm{d}^{\mathrm{m}} \mathrm{C}$ content by high-performance liquid chromatography (HPLC).
Jurkat cells were used because HPLC quantitation of DNA $\mathrm{d}^{\mathrm{m}} \mathrm{C}$ content requires larger amounts of DNA than can be obtained from IL-2-dependent cells. UV light was found to inhibit T cell DNA methylation in a dose-dependent manner (Figure 2A), with significant DNA methylation inhibition occurring by $45 \mathrm{~J} / \mathrm{m}^{2}$.

To test whether UV light also induces autoreactivity, PHA-activated CD4 $+\mathrm{T}$ cells were exposed to similar amounts of UV light, then challenged with autologous APC, using anti-CD3 as a positive control 
A

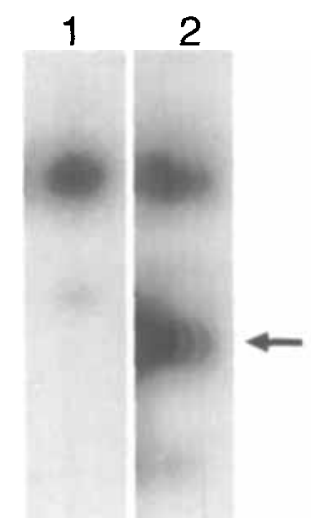

B

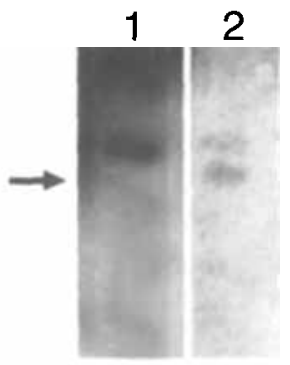

Figure 3. Southern analysis of transfectants. A, Sfi I and Pac I digest of DNA isolated from control TT18I cells (lane 1) and transfectants (lane 2) hybridized with a 1,400-bp fragment cut from the pEMO construct with $D p n \mathrm{I}$, containing approximately $500 \mathrm{bp}$ of the $5^{\prime}$ end of the CD18 insert (arrow) and $900 \mathrm{bp}$ of the retroviral backbone (lane 2). The arrow identifies the insert. B, DNA from control TT18I cells digested with $S f$ I, $P a c$ I, and $K p n$ I (lane 1) and transfectants digested with $S f$ I, Pac I, and Kpn I (lane 2) then hybridized with an 800 -bp fragment cut entirely from the CD18 insert with $B a m \mathrm{HI}$ and $B s t$ EII. Arrow identifies the migration of the 3.5-kb Kpn I fragment containing the CD18 cDNA, excised from the construct.

(Figure 2B). UV light induced autoreactivity similar to that induced by other DNA methylation inhibitors (2). Exposing a cloned TT-reactive T cell line to similar amounts of UV light, then challenging with APC with and without TT gave identical results (mean \pm SEM autoreactive response $9,829 \pm 1,065$ versus $20,587 \pm$ $2,498 \mathrm{cpm}$, untreated cells versus cells treated with 35 $\mathrm{J} / \mathrm{m}^{2}[P<0.02] ;$ antigen responses $24,232 \pm 965$ and $26,858 \pm 3,191$, untreated versus $\left.35 \mathrm{~J} / \mathrm{m}^{2}\right)$. An increase in LFA-1 expression (0.5-40 log green fluorescence units) was also seen on a subset of the $T$ cell clones exposed to amounts of UV light-inhibiting DNA methylation and inducing autoreactivity (Figures 2C and D). These experiments, coupled with our previous results, show that all 3 DNA methylation inhibitors can induce autoreactivity and increase LFA-1 expression.

To determine if LFA-1 overexpression could contribute to autoreactivity, we tested whether plasmidmediated LFA-1 overexpression could also induce autoreactivity. Cloned TT-reactive T cells (TT18I) were transfected simultaneously with CD11a and CD18 constructs, as described in Materials and Methods. Controls included untransfected cells and cells transfected with the parent CDM8 and pEMO vectors without inserts. Transfectants were selected by culturing in IL-2-

containing media with autologous APC but without TT. After $6-8$ weeks, a line grew out from the cells transfected with the constructs, but not from the controls. Identical results have been seen in 2 out of 2 repeats of this experiment, while lines could not be established using the parent expression vectors without inserts.

We next examined whether the selected cells had integrated the constructs. Figure $3 \mathrm{~A}$ shows a Southern blot, performed approximately 14 weeks after the transfection, in which DNA fragments from untransfected TT18I control cells and transfectants were hybridized with a 1,400-basepair fragment of the pEMO construct containing the $5^{\prime}$ end of the CD18 insert and a portion of the plasmid backbone. The experiment demonstrates genomic $\mathrm{CD} 18$ in both preparations, and stable integration of the construct in the transfectants, but not in the untransfected cells. Figure 3B shows a blot containing DNA from control cells and transfectants digested with $K p n$ I to excise the CD18 insert from the pEMO construct (14). The blot has been hybridized with a probe derived from an 800-bp fragment of the CD18 cDNA. Untransfected cells have a $3.8-\mathrm{kb}$ band representing genomic CD18, while the transfectants show a $3.5-\mathrm{kb}$ band corresponding to the CD18 insert in addition to the genomic CD18 band. In contrast to CD18, the CD1 la construct was not integrated (data not shown).

Flow cytometric analysis was used to examine LFA-1 expression on the transfected cells. In these experiments, cells undergoing selection by stimulation with APC alone were serially stained for CD11a and $\mathrm{CD} 18$, using $\mathrm{CD} 2$ or $\mathrm{CD} 3$ as reference markers. All electroporated clones initially showed decreased LFA-1, CD2, and CD3 expression. However, in those cells which grew through selection, a subset was detected which overexpressed both CD11a and CD18 $\sim 3$-fold relative to the lower-expressing subset. Figure 4 shows this subset using cells stained for CD11a. An identical subset was seen when cells were similarly stained for CD18 (data not shown). Using CD2 as a reference marker, we confirmed that the RFI of the population expressing lower amounts of LFA-1 had not changed from shortly after transfection, and that the population expressing greater amounts of LFA-1 was also increased relative to CD2. These experiments suggest that the integrated CD18 cDNA causes an $\sim 3$-fold increase in LFA-1 expression, relative to other surface markers and the control transfectants. A 2-fold increase in CD18 expression was also seen on a second, autoreactive transfected line.

The amount of antigen and number of APC 

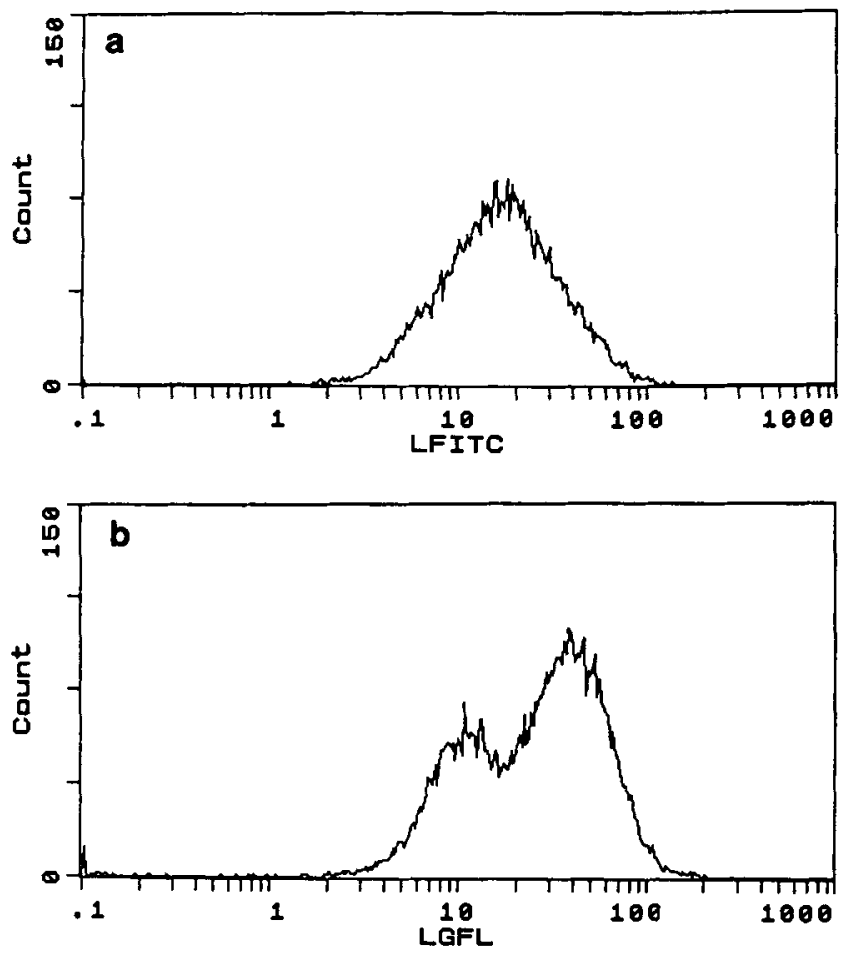

Figure 4. CD1 la expression in transfected TT18I cells. The transfectants described in Figure 3 were stained with anti-CD11a and fluorescein isothiocyanate (FITC)-conjugated goat anti-mouse Ig at A, 4 weeks and B, 16 weeks after electroporation, then analyzed using a Coulter ELITE flow cytometer. At 4 weeks, there is a single peak at 16 ; by 16 weeks, there are peaks at 12 and 40 . Values are the $\log$ FITC fluorescence (LFITC) and log green fluorescence (LGFL).

required for activation of the transfected and control cells was examined next. TT18I or TT18I transfectants were challenged with TT and a variable number of irradiated APC. The transfectants responded optimally to a lower number of APC compared with the untransfected cells, including numbers of APC insufficient to stimulate the untransfected cells (Figure 5A). Figure 5B shows a parallel experiment in which transfected and untransfected cells were challenged with increasing numbers of APC without TT. The transfectants are able to respond to autologous APC without added TT, although the magnitude of the autoreactive response is smaller than the antigen-induced response. Experiments performed using a second similarly transfected and selected line confirmed autoreactivity (mean \pm SEM $1,365 \pm 118$ versus $3,980 \pm 330 \mathrm{cpm}$, parent versus transfectant, $2 \times 10^{4} \mathrm{~T}$ cells and $10^{5}$ PBMC). This autoreactive response may be responsible for the continued growth of the transfected cells without antigen. Figure $5 \mathrm{C}$ compares the antigen re- quirements of transfected and control cells. Similar to the experiments studying the requirement for APC, the transfectants responded to lower concentrations of TT than did the untransfected cells, and even responded to amounts of TT that were not sufficient to stimulate untransfected cells.

Together, these experiments demonstrate that the transfected cells respond to stimuli insufficient to trigger a response in the untransfected cells, suggesting that the transfectants are capable of responding to otherwise-subthreshold stimulation signals, including those provided by APC without antigen. In these experiments, the magnitude of the optimal response by the transfectants is also greater than that observed with the untransfected cells. The reason for this is not clear, but it is possible that increased LFA-1 expression facilitates $T$ cell-APC interactions, leading to activation of a greater percentage of $\mathrm{T}$ cells.

Since experiments in the 5-azaC system had shown that the autoreactive response was more sensitive to anti-LFA-1 inhibition than was the antigen response, we next asked if the autoreactive response in the CD18 transfectants was also more sensitive to inhibition with anti-LFA-1 than was the antigen response (Figure 5D). Using a fixed number of $T$ cells and APC that simultaneously give significant and measurable antigen and autoreactive responses, antiCD1 la inhibited the autoreactive response by $74 \pm 6 \%$ (mean \pm SEM) and the TT response by $36 \pm 3 \%(P<$ 0.01 ). In this experiment, the autoreactive response was of greater magnitude than the antigen response. This is because it is important to use the same number of ligand-bearing APC and T cells for both the antigen and autoreactive responses to compare effects of the same amount of antibody. In contrast to the effect of antiCD11a-containing ascites, identical concentrations of mouse ascites containing a noninhibitory anti-CD11a (TA-1) had no significant effect on T cell activation (99.6 $\pm 4.2 \%$ of control, mean \pm SEM of 2 experiments).

These results support the idea that the autoreactive response is more sensitive to inhibition by the same amount of antibody than is the antigen response, similar to 5-azaC-treated cells. Together, these experiments suggest that LFA-1 overexpression mediated indirectly by treatment with DNA methylation inhibitors or directly by plasmid transfection is associated with $\mathrm{T}$ cell autoreactivity.

\section{DISCUSSION}

One goal of this laboratory is to determine how 5-azaC makes $T$ cells autoreactive. The many effects 
A.
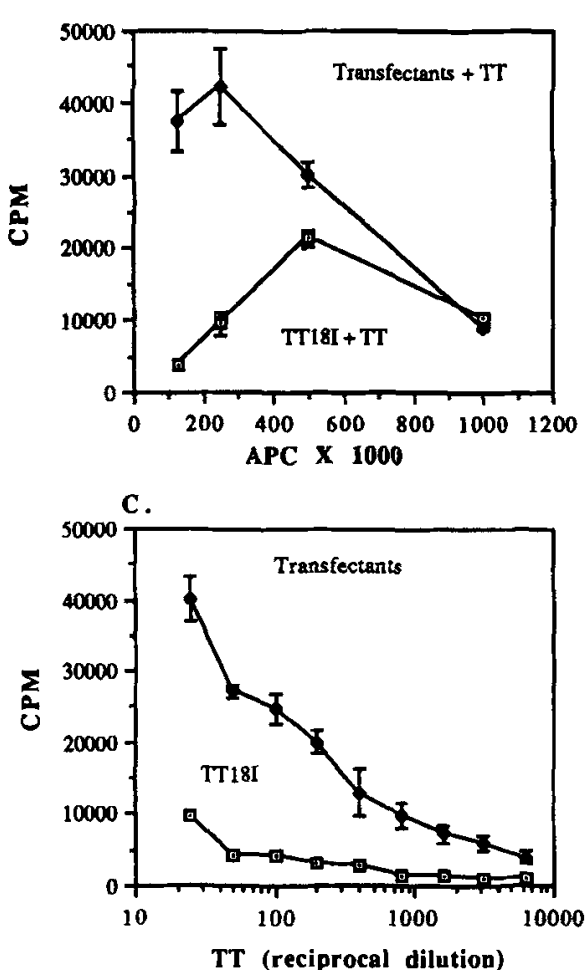

B.

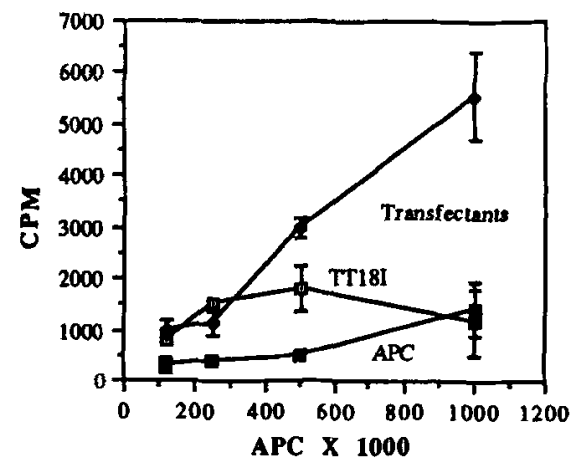

D.

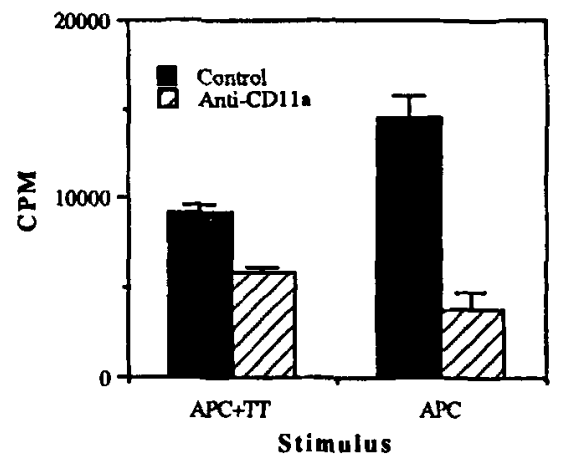

Figure 5. Activation requirements of control and transfected TT18I cells. A, Effect of transfection on antigen-presenting cell (APC) requirements for antigen-induced responses. TT181 or TT18I transfectants $\left(5 \times 10^{4}\right.$ cells) were challenged with tetanus toxoid (TT; diluted 1:50) and the indicated number of irradiated autologous APC. Proliferation was measured as described in Figure 2. Values are the mean \pm SEM of triplicate determinations. B, Effect of transfection on APC requirements for autoreactive responses. Transfected and untransfected TT18I cells $\left(5 \times 10^{4}\right)$ were challenged with increasing numbers of irradiated autologous APC without TT. ${ }^{3} \mathrm{H}$-thymidine incorporation by the irradiated APC alone is also shown $\left(P<0.02\right.$, transfected versus control at $\left.10^{6} \mathrm{APC}\right)$. C, Effect of transfection on antigen requirements. Transfected or control TT18I cells $(20,000)$ were cultured with $10^{5}$ irradiated autologous APC and the indicated dilutions of TT. Values are the mean \pm SEM of quadruplicate determinations. D, Effect of anti-lymphocyte function-associated antigen 1 on antigen reactivity and autoreactivity in transfected cells. Transfected TT18I cells $\left(5 \times 10^{4}\right)$ were challenged with $10^{6}$ autologous irradiated APC, with or without TT (diluted 1:50). Solid bars show cultures without added antibody; hatched bars show those with anti-CD11a $(1: 1,000)$.

of 5-azacytidine on cells (22) suggest multiple possible mechanisms by which autoreactivity may be induced. However, at concentrations inducing autoreactivity, the predominant effect is DNA methylation inhibition $(22,23)$. Therefore, it seems reasonable to propose that the autoreactivity is due to altered expression of one or more $\mathrm{T}$ cell genes regulated by DNA methylation. Previous experiments had examined $T$ cell clones for changes in gene expression induced by 5 -azaC. Twodimensional polyacrylamide gel electrophoresis of total cellular proteins and ${ }^{125} \mathrm{I}$-labeled membrane proteins were used to screen for new gene expression induced by 5 -azaC. However, no unique changes in cytoplasmic or cell surface polypeptides were observed (8). Similarly, immunoprecipitates of the T cell receptor (TCR) showed no changes in structure (8).
Flow cytometry was also used to examine alterations in the expression of $\mathrm{T}$ cell surface molecules following treatment with 5-azaC. No change was seen in the expression of $\mathrm{CD1}, 2,3,4,5,7,8,10,25,29,30,34$, $38,43,44,45 \mathrm{RA}, 45 \mathrm{RO}, 53,54,56,57,58$, w60, 69, 70, $73,4 \mathrm{~F} 2$, or TCR $\alpha / \beta(8)$.

More recently, an increase in CD28 has been observed in newly established clones, but this marker disappeared with time as reported by others (24), and was not re-expressed following 5-azaC treatment. However, the cells still became autoreactive, suggesting that CD28 was not likely to participate in the autoreactivity. CD6 up-regulation, which might contribute to autoreactivity, was demonstrated on 3 of 4 cloned lines. HLA-DR expression increased on 1 of 5 cloned lines, as did CD26, 46, and 48 (unpublished 
results). Each of these molecules has been implicated in $T$ cell activation (25-28), but the failure to alter expression on all clones raises questions about their role in inducing autoreactivity. Finally, more recent experiments have shown no alterations in $\mathrm{T}$ cell Fas expression induced by 5 -azaC (unpublished results).

Using a panel of 5 cloned $T$ cell lines, the most reproducible change induced by 5 -azaC was an increase in LFA-1 expression (8). Kinetic analysis revealed that CD11a expression usually began to increase 3 days after 5-azaC treatment and was maximal by day 4 , correlating with autoreactivity (8). A role for LFA-1 overexpression in autoreactivity was suggested by experiments in which small amounts of anti-LFA-1 were found to completely inhibit autoreactivity without affecting antigen reactivity (8). This is consistent with the interpretation that inhibiting the effects of the increased number of LFA-1 molecules would reverse the autoreactivity, suggesting that the increase in LFA-1 might cause the autoreactive response.

The current observations that PCA and UV light also induce autoreactivity and increase LFA-1 expression on $\mathrm{T}$ cell clones further support this association. It should be noted that earlier studies demonstrated that PCA also increases LFA-1 expression on murine CD4 + T cells (3). However, those studies were performed using polyclonal cells, so it was not clear whether PCA treatment was selected for cells with greater LFA-1 expression or directly increased LFA-1 expression. We have now confirmed the correlation between increased LFA-1 expression and autoreactivity using a cloned murine $\mathrm{T}$ cell line as well. The previous and present observations that 5-azaC, PCA, and UV light increase LFA-1 on human and murine T cell clones while inducing autoreactivity provide evidence for a direct effect of these agents on LFA-1 expression, and support the proposed correlation between increased LFA-1 expression and autoreactivity.

Transfection experiments tested the hypothesis that LFA-1 overexpression alone can contribute to autoreactivity. In these experiments, TT-reactive cells were transfected with cDNAs that encode LFA-1 subunits, and transfectants selected for autoreactivity. Autoreactive lines were established using this approach, and the line selected for study had stably incorporated the CD18 cDNA. These cells demonstrated increased LFA-1 expression, and the cells were able to respond to signals which were insufficient to activate untransfected cells, including APC without specific antigen. The autoreactive response was more sensitive to inhibition with anti-LFA-1 than was the antigen response, similar to the 5-azaC-treated cells. Together, these results are consistent with the interpretation that an increase in LFA-1, caused by overexpression of one of its subunits, permits $T$ cells to respond to normally subthreshold activation signals, either by providing additional stabilization to the TCR-MHC-antigen complex or by providing stronger transmembrane signals (29). The magnitude of the autoreactive response observed in the transfected cells is less, relative to the antigen response, than is the autoreactivity induced by DNA methylation inhibitors $(1,2)$. This may reflect the relatively smaller increase in LFA-1 achieved by transfection, compared to treatment with DNA methylation inhibitors, or possibly effects of DNA methylation inhibitors on other gene products, such as CD6. In contrast, the antigen response of the transfectants is significantly greater than the antigen response of the controls, presumably also due to LFA-1 overexpression.

The transfection experiments raise the question as to why the CD1la construct was not incorporated into the DNA. One explanation is that the transfection process in $\mathrm{T}$ cell clones is relatively inefficient, such that recombination events are rare, and incorporation of the CD18-pEMO construct, rather than the CD11aCDM8 construct, was by chance. It is also possible that $\mathrm{pEMO}$, the vector used to transfect the CD18 cDNA, may be more efficient in incorporating into human lymphocyte DNA than CDM8, which was used with CD1 la. This is supported by the recent successful stable transfection of human B cells with the same pEMO-CD18 construct (14).

Other interpretations of the transfection experiments were considered. It is possible that the transfection process, or incorporation of the vector backbone alone, contributed to the autoreactivity. However, these explanations are unlikely for 3 reasons. First, attempts to establish autoreactive lines using the parent expression vectors were unsuccessful. This indicates that the transfection process alone does not produce autoreactive cells through random insertion of a vector, and that the parent vectors alone are insufficient to produce autoreactivity. Second, the observation that the transfected cells respond to normally subthreshold stimulatory signals is consistent with known effects of LFA-1, which has previously been shown to stabilize low-affinity TCR interactions (30). Third, the autoreactivity could be preferentially reversed using small amounts of anti-LFA-1, consistent with the interpretation that inhibiting the function of the additional LFA-1 molecules reverses the auto- 
reactivity. It will be important to confirm these results by transfecting cells with a construct containing both the CD18 cDNA and a drug-resistance marker, selecting for the marker, then determining whether the transfectants overexpress LFA-1 and are autoreactive. These experiments would be more appropriately performed using murine cells, however, because autoreactivity could be confirmed by adoptive transfer experiments, looking for induction of autoimmunity similar to that caused by 5-azaC- and PCA-treated cells (3). This approach could also be used to test the effects of overexpressing other genes in $T$ cells.

The autoreactivity induced by LFA-1 overexpression has relevance to human lupus. As shown above and elsewhere (2), agents known to trigger lupus, such as PCA and UV light, increase $T$ cell LFA-1 expression and induce autoreactivity, and murine $T$ cells made autoreactive with 5 -azaC or PCA cause lupus in syngeneic mice (3). It is possible that the LFA-1 overexpression contributes to the lupuslike disease seen in this system. It is also interesting to note that the amount of UV light needed to inhibit DNA methylation and induce autoreactivity is approximately one-tenth that which causes a sunburn in Caucasians (31), supporting the hypothesis that UV light exposure in amounts frequently encountered by lupus patients could have an effect on their $T$ cells. Finally, we have reported that patients with idiopathic lupus have impaired T cell DNA methylation (5), and that patients with active lupus have an autoreactive $T$ cell subset identified by a relative overexpression of LFA-1, similar to 5-azaC- or PCA-treated cells (8). Together, these observations support a model in which LFA-1 expression is abnormally increased on CD4+ T cells, possibly by mechanisms involving DNA hypomethylation, and leading to $\mathrm{T}$ cell autoreactivity. The autoreactive $\mathrm{T}$ cells may then mediate a lupus-like autoimmune disease.

\section{ACKNOWLEDGMENTS}

The authors thank Drs. Tim Springer and James Wilson for their generous contributions of the CD1la and CD18 constructs, Drs. John Krauss, Michael Clarke, and Beverly Davidson for helpful discussions, Drs. David Fox and Blake Roessler for reviewing the manuscript, and Ms Beverly Saunders for her excellent secretarial assistance.

\section{REFERENCES}

1. Richardson B: Effect of an inhibitor of DNA methylation on $T$ cells. II. 5-azacytidine induces self-reactivity in antigen-specific T4+ cells. Hum Immunol 17:456-470, 1986
2. Cornacchia E, Golbus J, Maybaum J, Strahler J, Hanash S, Richardson B: Hydralazine and procainamide inhibit $\mathrm{T}$ cell DNA methylation and induce autoreactivity. J Immunol 140: 2197-2200, 1988

3. Quddus J, Johnson KJ, Gavalchin J, Amento EP, Chrisp CE, Yung RL, Richardson BC: Treating activated CD4+ $\mathrm{T}$ cells with either of two distinct DNA methyltransferase inhibitors, 5 -azacytidine or procainamide, is sufficient to cause a lupus-like disease in syngeneic mice. J Clin Invest 92:38-53, 1993

4. Lee SL, Chase PH: Drug-induced systemic lupus erythematosus: a critical review. Semin Arthritis Rheum 5:83-103, 1975

5. Richardson B, Scheinbart L, Strahler J, Gross L, Hanash S, Johnson M: Evidence for impaired T cell DNA methylation in systemic lupus erythematosus and rheumatoid arthritis. Arthritis Rheum 33:1665-1673, 1990

6. Cedar H: DNA methylation and gene expression, DNA Methylation: Biochemistry and Biological Significance. Edited by A Razin, H Cedar, AD Riggs. New York, Springer-Verlag, 1984

7. Richardson B, Kahn L, Lovett EJ, Hudson J: Effect of an inhibitor of DNA methylation on T cells. I. 5-azacytidine induces $\mathrm{T} 4$ expression on $\mathrm{T} 8+\mathrm{T}$ cells. $\mathrm{J}$ Immunol 137:35-39, 1986

8. Richardson BC, Strahler JR, Pivirotto TS, Quddus J, Bayliss GE, Gross LA, O'Rourke KS, Powers D, Hanash SM, Johnson MA: Phenotypic and functional similarities between 5azacytidine-treated $\mathrm{T}$ cells and a $\mathrm{T}$ cell subset in patients with active systemic lupus erythematosus. Arthritis Rheum 35:647662,1992

9. Rabin H, Hopkins RF, Ruscetti FW, Neubauer RH, Brown RL, Kawakami TG: Spontaneous release of a factor with properties of $\mathrm{T}$ cell growth factor from a continuous line of primate tumor T cells. J Immunol 127:1852-1856, 1981

10. Richardson BC, Liebling MR, Hudson JL: CD4+ cells treated with DNA methylation inhibitors induce autologous $B$ cell differentiation. Clin Immunol Immunopathol 55:368-381, 1990

11. Sanchez-Madrid F, Krensky AM, Ware CF, Robbins E, Strominger JL, Burakoff SJ, Springer TA: Three distinct antigens associated with human T-lymphocyte-mediated cytolysis: LFA-1, LFA-2, and LFA-3. Proc Natl Acad Sci U S A 79:74897493,1982

12. LeBien TW, Bradley JG, Koller B: Preliminary structural characterization of the leukocyte cell surface molecule recognized by monoclonal antibody TA-1. J Immunol 130:1833-1836, 1983

13. Seed B: An LFA-3 cDNA encodes a phospholipid-linked membrane protein homologous to its receptor CD2. Nature 329:840842,1987

14. Wilson JM, Ping AJ, Krauss JC, Mayo-Bond L, Rogers CE, Anderson DC, Todd RF: Correction of CD18-deficient lymphocytes by retrovirus-mediated gene transfer. Science $248: 1413$ 1416,1990

15. Golbus J, Palella TD, Richardson BC: Quantitative changes in T cell DNA methylation occur during differentiation and ageing. Eur J Immunol 20:1869-1872, 1990

16. Friedman S: The inhibition of DNA(cytosine-5) methylases by 5-azacytidine: the effect of azacytosine-containing DNA. Mol Pharmacol 19:314-320, 1981

17. Scheinbart LS, Johnson MA, Gross LA, Edelstein SR, Richardson BC: Procainamide inhibits DNA methyltransferase in a human $T$ cell line. J Rheumatol 18:530-534, 1991

18. Lieberman MW, Beach LR, Palmiter RD: Ultraviolet radiationinduced metallothionein-I gene activation is associated with extensive DNA demethylation. Cell 35:207-214, 1983

19. Wagner JR, Hu C-C, Ames BN: Endogenous oxidative damage of deoxycytidine in DNA. Proc Natl Acad Sci U S A 89:33803384,1992

20. Jones PA: Gene activation by 5-azacytidine, DNA Methylation: 
Biochemistry and Biological Significance. Edited by A Razin, H Cedar, AD Riggs. New York, Springer-Verlag, 1984

21. Sanders ME, Makgoba MW, Sharrow SO, Stephany D, Springer TA, Young HA, Shaw S: Human memory T lymphocytes express increased levels of three cell adhesion molecules (LFA-3, CD2, and LFA-1) and three other molecules (UCHL1, CDw29, and Pgp-1) and have enhanced IFN- $\gamma$ production. J Immunol 140:1401-1407, 1988

22. Cihak A: Biological effects of 5-azacytidine in eukaryotes. Oncology 30:405-422, 1974

23. Glazer RI, Hartman KD: The comparative effects of 5-azacytidine and dihydro-5-azacytidine on $4 \mathrm{~S}$ and $5 \mathrm{~S}$ nuclear RNA. Mol Pharmacol 17:250-255, 1980

24. Testi R, Lanier LL: Functional expression of CD28 on T cell antigen receptor gamma/delta-bearing $T$ lymphocytes. Eur $J$ Immunol 19:185-188, 1989

25. Gangemi RM, Swack JA, Gaviria DM, Romain PL: Anti-T12, an anti-CD6 monoclonal antibody, can activate human $\mathrm{T}$ lymphocytes. J Immunol 143:2439-2447, 1989

26. Dang NH, Torimoto $Y$, Sugita K, Daley JF, Schow P, Prado C, Schlossman SF, Morimoto C: Cell surface modulation of CD26 by anti-1F7 monoclonal antibody: analysis of surface expression and human T cell activation. J Immunol 145:3963-3971, 1990

27. King PD, Batchelor AH, Lawlor P, Katz DR: The role of CD44, CD45, CD45RO, CD46 and CD55 as potential anti-adhesion molecules involved in the binding of human tonsillar $\mathrm{T}$ cells to phorbol 12-myristate 13-acetate-differentiated U-937 cells. Eur J Immunol 20:363-368, 1990

28. Yokoyama S, Stauton D, Fisher R, Amiot M, Fortin JD, Thorley-Lawson DA: Expression of the blast-1 activation/ adhesion moleculae and its identification as CD48. J Immunol 146:2192-2200, 1991

29. Wacholtz MC, Patel SS, Lipsky PE: Leukocyte functionassociated antigen 1 is an activation molecule for human $T$ cells. J Exp Med 170:431-448, 1989

30. Altmann DM, Hogg N, Trowsdale J, Wilkinson D: Cotransfection of ICAM-1 and HLA-DR reconstitutes human antigenpresenting cell function in mouse L cells. Nature 338:512-514, 1989

31. Cooper KD, Androphy EJ, Lowy D, Katz SI: Antigen presentation and $\mathrm{T}$-cell activation in epidermodysplasia verruciformis. J Invest Dermatol 94:769-776, 1990 